\title{
The effects of scopolamine on proactive interference and spatial delayed matching-to-sample performance in rats
}

\author{
H. L. ROITBLAT, HEIDI E. HARLEY, and DAVID A. HELWEG \\ University of Hawaii at Manoa, Honolulu, Hawaii
}

\begin{abstract}
Rats were trained in a three-alternative spatial delayed matching-to-sample task in which the samples were rewarded forced choices of one arm of a three-arm starburst maze and retention was indicated by returning to that arm following a delay or retention interval. If the rat made an error on its first free choice of a trial, the chosen arm was blocked off and the rat was allowed a second choice between the remaining two arms. In two experiments, we investigated rats' performance following presession treatment with scopolamine hydrobromide or following one of several control treatments presented in a repeated-measures design. In the first experiment, rats were given $0.1-\mathrm{mg} / \mathrm{kg}$ scopolamine hydrobromide, $0.1-\mathrm{mg} / \mathrm{kg}$ scopolamine methylbromide, or an equal volume of saline vehicle. In the second experiment, we tested new groups of rats under the same drug conditions as in Experiment 1 and in an additional condition without drugs, but with a longer retention interval. Experimental sessions were separated from one another by at least 1 rest day and $1 \mathrm{drug}$-free short-delay reminder session. Choice accuracy was lower following injections of scopolamine hydrobromide relative to the other conditions. Proactive interference, in the form of intrusions by previous samples or intrusions of the previous choices, however, was unaffected by drug treatment. These results suggest that scopolamine interferes with choice accuracy by decreasing the retention or encoding of information about the location of the sample, rather than by decreasing the animal's ability to differentiate the responses made on one trial from those made on the previous trial.
\end{abstract}

The cholinergic systems of the brain have long been implicated in memory function and dysfunction. In particular, administration of scopolamine (a muscarinic receptor blocker) causes impairment in human memory (e.g., see Crow \& Grove-White, 1973; Deutsch, 1983; Drachman \& Leavitt, 1974; Parrott, 1987). The peripherally active methscopolamine, on the other hand, does not produce these effects (Drachman \& Leavitt, 1974). Several of the effects of scopolamine on human memory have been reviewed by Kopelman (1986).

The administration of anticholinergic agents has also been found to affect animal memory processes (Dunnett, 1985; Heise, Hrabich, Lilie, \& Martin, 1975; Heise \& Hudson, 1985; Huston \& Aggleton, 1987; Spencer, Pontecorvo, \& Heise, 1985; Viscardi \& Heise, 1986; see Rawlins, 1985, for a review). Some experiments have found that scopolamine interferes with working memory in the radial maze (e.g., Ammassari-Teule \& Caprioli, 1985; Eckerman, Gordon, Edwards, MacPhail, \& Gage, 1979; Godding, Rush, \& Beatty, 1982; Okaichi \& Jarrard, 1982; Rauch \& Raskin, 1984), but not with reference memory (Beatty \& Bierley, 1985; Levy, Kluge, \& Elsmore, 1983; Wirsching, Beninger, Jhamandas, Boeg-

This research was supported by National Science Foundation Grant BNS 85-05681. Request reprints from Herbert L. Roitblat, Department of Psychology, University of Hawaii at Manoa, 2430 Campus Road, Honolulu, HI 96822, or via electronic mail to herbert@uhccux.bitnet. man, \& El-Defrawy, 1984; see Okaichi \& Jarrard, 1982, for a review). However, the mechanism by which working memory is affected is not clear.

Roitblat and Harley (1988) investigated rats' performance in a three-alternative spatial delayed matching-tosample (SDMTS) task that allows the investigation of the means by which cholinergic agents affect memory (see Rawlins \& Olton, 1982, for a related procedure). They found evidence for separable proactive interference effects. Choice accuracy improved with increasing intertrial interval (ITI) durations and declined with increasing retention-interval durations. The choice made on one trial was affected by the sample presented or the choice made on the previous trial, and the degree to which these prior events influenced responding decreased as the ITI increased. In addition, choice accuracy was higher when the sample from one trial matched the sample from the previous trial and lower when the two successive samples did not match (see also Grant, 1981). The effects of ITI duration and successive samples were mediated by separate mechanisms, however, because choice accuracy improved with increasing ITI durations whether or not the previous sample matched the later sample. Long ITI durations increased choice accuracy, in other words, even if there was no competition from the memory of the previous trial (see also Roitblat \& Scopatz, 1983).

These results are inconsistent with a single proactive interference mechanism based on competition. Instead, 
they imply the existence of two separable proactive interference effects, one mediated by competition from the memory of the previous trial and the other mediated by encoding deficits resulting from closely spaced trials. Short ITI durations allow additional competition from the preceding trial and interfere with encoding sample information from the current trial.

The separable proactive interference effects suggest two sources of errors in delayed conditional discrimination tasks such as SDMTS. An animal could make errors either because it has an inadequate memory of the current trial or because the memory from the preceding trial competes with the memory from the current trial. For ease of exposition, these two hypotheses can be called the encodingfailure hypothesis and the temporal discrimination hypothesis. According to the temporal discrimination hypothesis, the animal makes an error because it fails to discriminate the temporal order of recent trials. The remaining memory representation of the previous trial happens to be momentarily more salient (stronger, clearer, more familiar, etc.) than the memory representation of the sample from the current trial. According to the encoding-failure hypothesis, the animal makes an error because the representation of the sample from the current trial is too "weak" (or obscure or unfamiliar) to yield a correct choice independent of the strengths of any other representations that happen to be available. The disruptive effects of scopolamine on animal memory have virtually all been demonstrated in tasks that conflate these two sources of error.

When there are only two response alternatives, an animal can make only one kind of error when it fails to make the correct choice. Therefore, it is not possible to discriminate among these sources of error. Experiments with radial mazes allow more response alternatives, but the nature of this task (the fact that only one trial is typically presented per day, and the task's requirement that the animal avoid arms it remembers having entered) also makes discriminating between these hypotheses difficult.

In this experiment, we provided three response alternatives and allowed animals to make a second choice following a first-choice error. An animal performing this task can make either of two kinds of error. It can select one of three maze arms visited on the previous trial (assuming that a different arm is correct), thus indicating an intrusion or perseveration error. Alternatively, it can err by selecting an arm that was visited neither on the previous trial nor on the present trial (a nonvisit error), thus indicating forgetting of the sample location. Given the frequencies of intrusions and nonvisit errors, one can estimate the relative importance of intrusions and encoding deficits as sources of errors in the SDMTS task.

\section{EXPERIMENT 1}

In the first experiment, rats already experienced in the SDMTS task were tested in a repeated-measures design with one of three drug conditions. In one condition (Scop), the rats received i.p. injections of the centrally active anticholinergic scopolamine hydrobromide. In the second condition (MScop), they received scopolamine methylbromide, which does not cross the blood-brain barrier in substantial amounts and apparently does not interfere with memory processing (Drachman \& Leavitt, 1974), although its effects on, for example, motor processing, are understood to be similar to the periperhal effects of scopolamine. In the third condition (Saline), the rats received equal-volume injections of saline vehicle to control for the effects of injection.

\section{Method}

Subjects. The subjects were 6 Sprague-Dawley rats obtained from Simonsen Laboratories in Gilroy, CA. All subjects were experienced in the SDMTS task, having served in a previous experiment (Roitblat \& Harley, 1988) or in a similar study and in pilot experiments during which the appropriate doses of scopolamine were determined. The subjects were approximately 12 months old at the start of training.

The rats were housed in individual cages under a 14:10 h light:dark regimen with free access to water. They were maintained at approximately $85 \%$ of their free-feeding weights by daily rations of Purina Rat Chow. Criterion weights were allowed to increase during the course of the experiment by comparing the food-deprived animals with a previously investigated nondeprived control group.

Apparatus. The rats were trained and tested in the same elevated three-arm starburst maze used by Roitblat and Harley (1988). This apparatus consists of a start compartment, a stem, a roughly triangular choice area, and three goal arms. The start compartment and goal arms were each separated from the rest of the apparatus by manually operated guillotine doors. A food cup was located at the end of each goal arm. A screen was used to separate the sample reward from the choice reward. Sessions were conducted in a welllighted room containing a window and a door at opposite ends of the room, tables with operant chambers, a stainless steel counter and sink, and other odds and ends typically found in an animal laboratory.

Procedure. Each trial consisted of a forced-entry sample run followed by either one or two choice runs. Before the sample run, all the doors of the maze were closed and the food cup in the goal compartment of one arm (selected according to a prearranged random schedule) was baited with one whole Froot Loop (a sweetened, fruit-flavored breakfast cereal manufactured by Kelloggs, weighing approximately $210 \mathrm{mg}$ each) and a piece of Froot Loop. The whole Froot Loop, or choice reward, was placed beneath a wire screen where it could be seen and smelled by the animal, but not eaten. The piece of Froot Loop, or sample reward, which varied somewhat in size between one-fourth and one-third of a whole Froot Loop, was placed on top of the screen. The rat was then placed in the start compartment with the door closed. The door to the baited goal arm was opened and the rat was released to run along the stem and into the baited goal arm. The door to the baited goal arm was closed when the rat passed it. The rat was returned to the start compartment immediately after it finished eating the Froot Loop piece from the food cup. The screen was then removed from the baited goal arm, all the doors to the choice arms were raised, and the animal was allowed a free choice among the three arms. Following correct choices, the animal was allowed to eat the whole Froot Loop choice reward. If the first choice was an error, then the door to the erroneously entered arm was closed, and the rat was returned to the start compartment. It was then allowed a second choice between the remaining two arms. The animal was also allowed to eat the Froot Loop following correct second choices. Second-choice errors ended the trial. The minimum ITI was $30 \mathrm{sec}$. The rats were 
transported to the experimental room from their quarters in their home cages, where they spent the ITIs.

Each rat was tested once in random order under each of three conditions. Under Condition Scop, the rat received a $0.1-\mathrm{mg} / \mathrm{kg}$ injection of scopolamine hydrobromide. Under Condition MScop, the rat received a $0.1-\mathrm{mg} / \mathrm{kg}$ injection of scopolamine methylbromide. Under Condition Saline, the rat received an equivalent volume of saline control, but no anticholinergic agents. All drugs were administered i.p. in normal saline $(0.1 \mathrm{mg} / \mathrm{ml})$, approximately $15 \mathrm{~min}$ prior to the start of the session. Sessions lasted approximately $2 \mathrm{~h}$. Drugs were obtained from Sigma Biochemicals, St. Louis, MO.

Each drug session was preceded by a short-delay "reminder" session. The procedure during the reminder session was identical to that used during training. No drugs were given. The delay between the sample run and the choice run was as short as practical $(5-7 \mathrm{sec})$. Each reminder session consisted of 24 trials. Reminder sessions ensured that the rats received reminders of the SDMTS procedure and were able to perform the task prior to any drug treatment. Each drug session was also followed by at least 1 day on which no trials or drugs were given. These sessions also ensured that the drugs had ample opportunity to be eliminated prior to any other drug treatment.

Each drug session contained 49 trials. The retention interval between the time at which the rat finished eating the piece of Froot Loop and the time it was released from the start box was about $60 \mathrm{sec}$. The ITI was $30 \mathrm{sec}$.

The experimenter testing the rats did not know which drug the animals had received (drug vials were labeled by a code letter), but could not actually be blind to the drug condition because of the noticeable behavioral effects of scopolamine.

\section{Results}

Figure 1 shows the choice accuracy of the three groups under each drug condition. Choice accuracy under the Scop condition was significantly below that for the other conditions $[F(2,10)=4.77, p<.05]$. Figure 1 also shows the accuracy of second choices following firstchoice errors. Second-choice accuracy also differed significantly among the three drug conditions, with accuracy being the poorest in the Scop condition $[F(2,10)=10.46$, $p<.01]$.

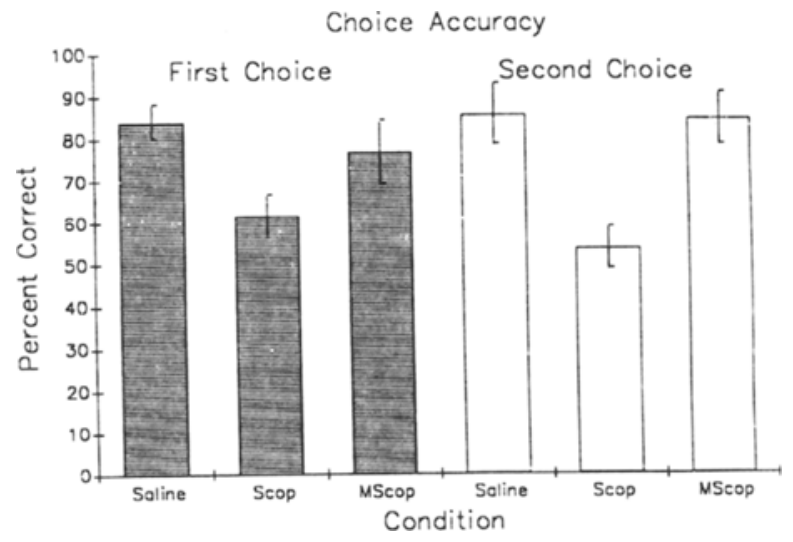

Figure 1. Mean choice accuracy under each drug condition in Experiment 1. Choice accuracy under the Scop condition was signifcantly below that for the other conditions. Scop $=$ presession treatment with scopolamine hydrobromide, MScop = presession treatment with scopolamine methylbromide, Saline $=$ presession treatment with saline vehicle.

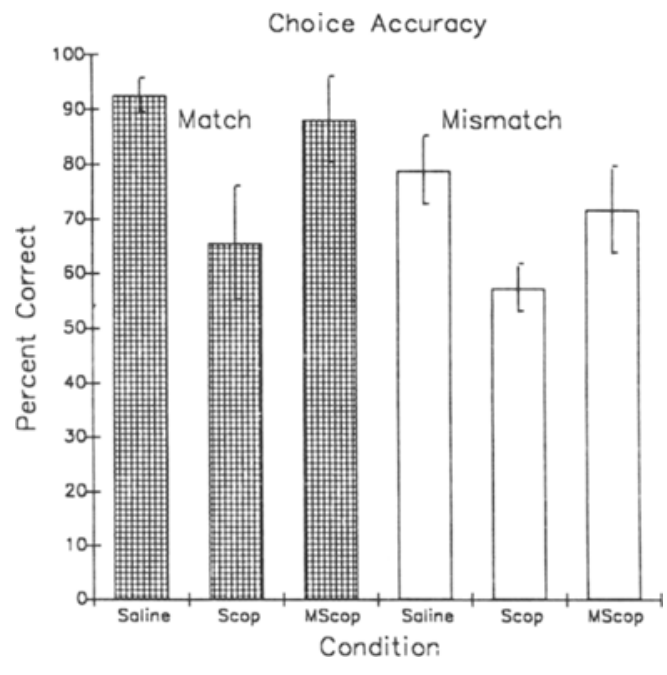

Figure 2. Mean choice accuracy as a function of drug condition and of agreement between the presample and the sample in Experiment 1. Choice accuracy was lower when the samples did not agree, but the effects of intrusions were roughly equivalent in the three drug conditions. Match indicates that the sample from the preceding trial was a forced entry to the same arm that served as the sample on the succeeding trial. Mismatch means that the sample from the preceding trial was one of the distractors on the succeeding trial.

We assessed the degree of intrusions from one trial to the next using the phi statistic. Phi is a measure of nonindependence or association between two nominal variables (see Roitblat \& Scopatz, 1983, for a discussion of phi). Phi is a function of chi-square, adjusted for differences in the number of observations. High values of chisquare, and hence of phi, indicate that two variables jointly determine the frequency of conjoint observations. Low values of chi-square, and hence of phi, indicate that two variables independently influence the frequency of conjoint observations. The phi values displayed in Figure 2 were computed from two $(3 \times 3)$ tables at each combination of ITI and retention-interval duration. One table contains the frequencies with which each choice was made following each presample. The presample is the sample from the immediately preceding trial. This table thus indicates the degree to which the sample appearing on one trial influenced the choice made on the next. The phi value for this table is, therefore, a measure of the presampleintrusion proactive interference effect. The second table contains the frequency with which each choice was made following each prechoice. The prechoice is the choice from the immediately preceding trial. This table thus indicates the degree to which the choice made on one trial influenced the choice made on the next.

Although the phi values representing the association between the presample and the choice and between the prechoice and the choice were both different from 0 $[F(1,5)=106.55, p<.001$, and $F(1,5)=87.57$, $p<.001$, respectively], the phi values did not differ significantly among the drug conditions (both $F \mathrm{~s}<1.0$ ). The drug condition had no effect on the magnitude of the 
association either between the presample and the choice or between the prechoice and the choice.

Figure 2 shows choice accuracy when the sample from the previous trial either matched or did not match the sample from the next trial. Intrusions in the form of lower choice accuracy are expected to occur when the sample from the previous trial is different from the sample on the current trial to the extent that the two memories compete to control performance. As Figure 2 shows, choice accuracy was lower when the samples did not agree $[F(1,5)=10.5, p<.05]$ than when they did agree. Consistent with the earlier analyses, choice accuracy was also seen to differ among the three drug conditions $[F(2,10)=$ $6.8, p<.05]$, but the effects of intrusions were roughly equivalent in the three drug conditions, as shown by the failure of the match $\times$ drug interaction to reach significance $(F<1.0)$.

\section{Discussion}

The results of this experiment are entirely consistent with the results of previous studies of the SDMTS task (Roitblat \& Harley, 1988). During the control sessions with scopolamine methylbromide or saline, the animals were able to return to the site of the sample reward with consistent, high accuracy (chance is $33 \%$ ). When their first choice was an error, they were more accurate than chance $(50 \%)$ in correctly making their second choices. Also similar to previous studies, the events of the previous trial-that is, the presample and the prechoice-had small but significant effects on the animals' choices on the next trial. In particular, choice accuracy was higher when the sample from the previous trial (the presample) agreed with or matched the sample from the next trial than when they did not match. These findings thus illustrate the presence of intrusions as at least one of the factors that controlled the animals' responding.

Consistent with a number of other studies of the effects of anticholinergics on memory, this experiment also demonstrated that pretreatment with a centrally active form of scopolamine interfered with accurate memory performance. The accuracy of both first and second choices was lower following an injection of scopolamine than under the other conditions, but the strength of the association between the events of one trial and the choice made on the next was unaffected by the drug treatment. Scopolamine hydrobromide lowered choice accuracy without either increasing or decreasing intrusions from the previous trial. Under all three drug conditions, choice accuracy was higher when the sample from one trial matched the sample presented on the previous trial than when they did not match, but the size of this difference was not affected by the drug treatment. The effects of intertrial sample agreement and the significantly nonzero phi values for the association between presample and choice and between prechoice and choice clearly show that intrusions played a role in determining choice accuracy, but this role was not affected by the drug treatments.

\section{EXPERIMENT 2}

The results of Experiment 1 indicate that scopolamine hydrobromide affected choice accuracy without affecting intrusions from the events of previous trials. The rats studied in that experiment had previously been exposed to the drugs used in the study and it is possible that their prior drug experience had somehow modified their reaction to the drug (e.g. , through some process akin to habituation or through some physiological adjustment to prior experience with scopolamine). Experiment 2 was conducted with a drug-naive group of animals in order to determine whether the results of Experiment 1 were peculiar to drug-experienced animals. Experiment 2 also included a fourth treatment condition to determine the comparability of the scopolamine-induced memory deficit with that obtained following longer delays. The logic of this fourth treatment is this. The memory trace resulting from a sample run presumably decreases gradually as time since the sample run increases (see Roitblat, 1980, 1984a, $1984 \mathrm{~b}, 1987$ ). If the effect of scopolamine is also to interfere with encoding, presumably by limiting the strength of the resulting memory trace, then the effects of scopolamine should be similar to those observed following a long retention interval.

Alternatively, intertrial proactive interference effects in rat delayed alternation performance have been found to be more prominent after a long than after a short (40vs. 0-sec) retention interval (Grant, 1981). Although the retention interval used in Experiment 1 was longer than the long interval used by Grant, it is possible that a longer interval in this task will also strengthen the proactive interference effect. On the basis of pilot testing, we selected a retention interval that could be expected to produce levels of first-choice accuracy similar to those obtained with scopolamine and a 60 -sec retention interval. This condition provides an interesting comparison in that similar levels of choice accuracy and higher levels of intrusions are predicted to occur with this longer delay than with scopolamine and the shorter delay. This condition thus has the potential to demonstrate separable sources of errors in the SDMTS task if increasing the retention interval introduces a pattern of errors that is different from that produced by anticholinergic treatment.

\section{Method}

Subjects. The subjects were 9 Sprague-Dawley rats obtained from Simonsen Laboratories in Gilroy, CA. Three of the rats were approximately 3 months of age when they arrived in the laboratory. Four rats were retired breeders and were approximately 12 months old on arrival. These animals were experimentally naive at the start of the present study. The remaining 2 rats had served in previous SDMTS experiments (Roitblat \& Harley, 1988) and were approximately 22 months of age at the start of the experiment. The rats were maintained under conditions identical to those used in Experiment 1. Although the rats differed in age, age was not a primary variable of interest in the present study.

Apparatus. The apparatus was identical to that used in Experiment 1 . 
Procedure. The experimentally naive rats were first trained to perform the SDMTS task in 24-trial daily sessions. The structure of these trials was identical to that used in Experiment 1 and in the experiment reported by Roitblat and Harley (1988), except that the retention interval between the end of the sample run and the start of the first choice run was about 5-6 sec.

Following acquisition training, the rats were tested at several retention-interval delays between the sample event and the choice phase before beginning the drug treatments. Each rat was then tested once in random order under each of four conditions. Under Condition Scop, the rat received a $0.1-\mathrm{mg} / \mathrm{kg}$ injection of scopolamine hydrobromide. Under Condition MScop, the rat received a $0.1-\mathrm{mg} / \mathrm{kg}$ injection of scopolamine methylbromide. Under Condition Saline, the rat received an equivalent volume of saline vehicle. Under Condition NoDrug, the rat received no injection. Drugs were administered in a manner identical to that used in Experiment 1.

Each experimental session (including the NoDrug condition) was preceded by a 24-trial, short-delay session (identical in procedure to the training sessions) and was followed by at least 1 day on which no trials or drugs were given. Each dng session contained 49 trials. The retention interval between the time when the rat finished eating the piece of Froot Loop from the sample run and the time it was released from the startbox for its first choice run was about $60 \mathrm{sec}$. The ITI was $30 \mathrm{sec}$. NoDrug sessions also contained 49 trials, but with a $120-\mathrm{sec}$ retention interval. Pilot testing indicated that approximately equivalent levels of choice accuracy could be expected in Condition Scop and Condition NoDrug with these retention-interval durations.

\section{Results}

The age groups did not differ significantly in any of the analyses; therefore, the data combine the age groups. All rats were trained to a criterion of $90 \%$ correct performance on two successive sessions.

Figure 3 shows the choice accuracy of the subjects under each treatment condition. Choice accuracy differed among the four treatment conditions $[F(3,24)=3.39$, $p<.05]$. Planned comparisons revealed that choice ac-

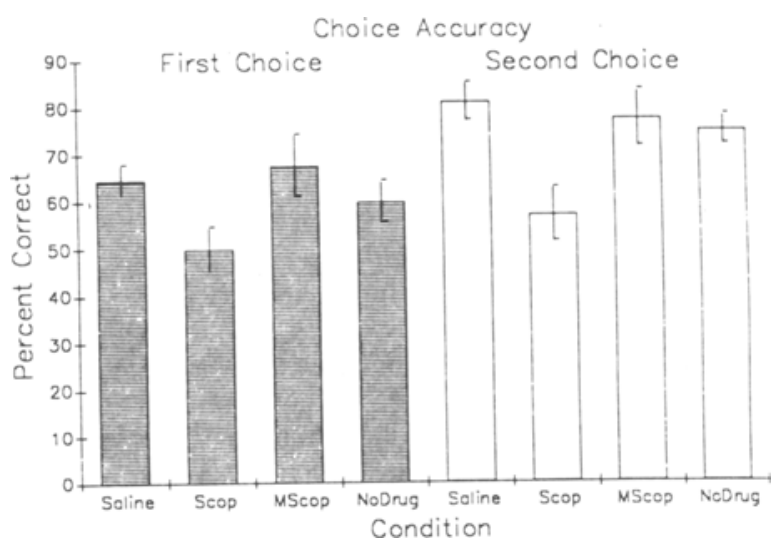

Figure 3. Mean choice accuracy under each drug condition in Experiment 2. First-choice accuracy under the Scop condition was significantly below that for the Saline and MScop conditions. Secondchoice accuracy under Scop was poorer than under the other three conditions. Scop $=15$-min presession treatment with scopolamine hydrobromide, MScop $=15$-min presession treatment with scopolamine methylbromide, Saline $=$ pretreatment with saline vehicle, NoDrug $=$ no injection but increased retention-interval duration.

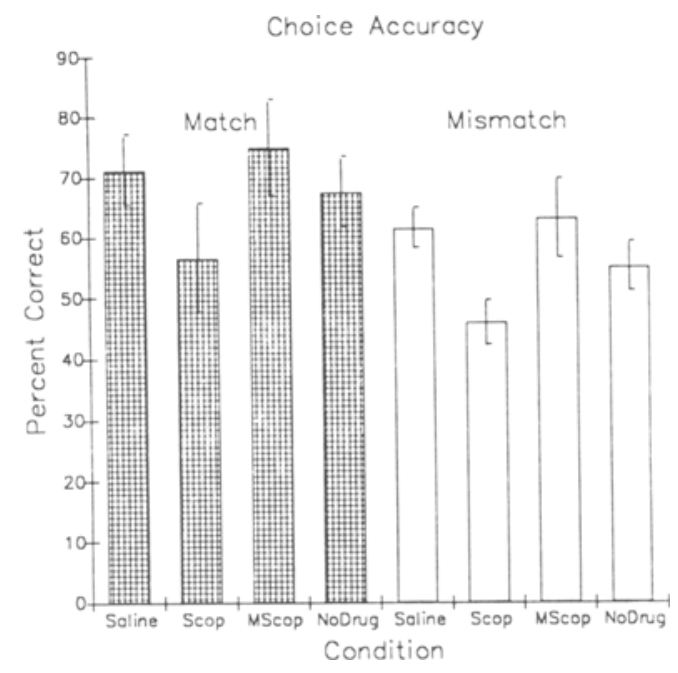

Figure 4. Mean choice accuracy as a function of drug condition and of agreement between the presample and the sample in Experiment 2. Choice accuracy was higher when the sample from one trial matched the sample from the previous trial. Match indicates that the sample from the preceding trial was a forced entry to the same arm that served as the sample on the succeeding trial. Mismatch means that the sample from the preceding trial was one of the distractors on the succeeding trial.

curacy under the Scop condition was significantly below that for the Saline and MScop conditions $[F(1,24)=9.89$, $p<.01]$, but was not less than that under the NoDrug condition $[F(1,24)=2.81, p>.05]$, which was not significantly different from the Saline and MScop conditions $[F(1,24)=1.46, p>.05]$. Figure 3 also shows the accuracy of second choices following first-choice errors. Second-choice accuracy differed among the drug conditions $[F(3,24)=5.21, p<.05]$. Planned comparisons revealed that second-choice accuracy under Scop was poorer than under the other three conditions $[F(1,24)=$ $14.81, p<.01]$, which did not differ among themselves.

We also computed the strength of the relationship between the events of one trial and the choice made on the next in terms of phi values. As in the first experiment, both the presample and the prechoice had nonzero phi values $[F(1,8)=134.7, p<.001$, and $F(1,8)=207.38$, $p<.001$, respectively], but neither the association between the presample and the choice $(F<1.0)$ nor the association between the prechoice and the choice $[F(3,24)=1.21, p>.1]$ varied among the conditions. These associations show the degree to which the memory for the sample event or the choice from the previous trial influenced the choice made on the next trial. As in Experiment 1 , the drug condition did not affect the strength of the relationship between the sample from the previous trial (the presample) and the choice on the next, or the relationship between the choice from the previous trial (the prechoice) and the choice on the next.

Figure 4 displays choice accuracy as a function of drug condition and of the agreement between the presample and the sample. Choice accuracy was higher when the sam- 
ple from one trial matched the sample from the previous trial than when they did not match $[F(1,8)=10.98$, $p<.05]$.

\section{Discussion}

The results of Experiment 2 replicate, with naive animals, the results of Experiment 1 . Injections of centrally active scopolamine interfered with choice accuracy without increasing the degree of intrusions by the events of the previous trial.

In general, the effects of scopolamine hydrobromide were similar to, though more pronounced than, those obtained with an increased delay between the sample run and the free-choice run. The primary difference between the two treatments was that scopolamine, tested with a 60 -sec retention interval, was more disruptive to secondchoice accuracy than was simply waiting during a $120-\mathrm{sec}$ retention interval with no drug. In other ways, including the decrease in first-choice accuracy, the administration of scopolamine had effects that were similar to increasing the retention interval. It seems reasonable, therefore, to infer that scopolamine exerted its effect by reducing the strength or salience of the sample run at the time of the choice run.

\section{GENERAL DISCUSSION}

Consistent with a number of other studies of the effects of anticholinergics on memory, these experiments also demonstrated that treatment with a centrally active form of scopolamine has a deleterious effect on memory performance. Choice accuracy was lower when the rats were pretreated with scopolamine $15 \mathrm{~min}$ before testing. Furthermore, treatment with scopolamine not only decreased the accuracy of first choices, but also decreased the accuracy of second choices following first-choice errors. In contrast, scopolamine had no effect on the degree of intrusions from the events of previous trials. Both experiments found that choice accuracy was higher when the sample from the current trial matched the sample presented on the previous trial, but the difference in choice accuracy between matching and nonmatching pairs of trials was unaffected by the drug condition.

Other studies have found that scopolamine increases perseveration (e.g., Heise et al., 1975), but studies of human list-learning have generally found no increase in intrusions of members of one list during recall of another following scopolamine treatment. The animal experiments that have found an increase in perseveration generally involved only two response alternatives; therefore, one cannot know whether an increase in errors is due to repeating the previously made choice or to forgetting the correct current choice. Both hypothetical processes yield exactly the same prediction, a return to the incorrect arm visited on the previous trial. In contrast, in the present experiments, we employed three choice alternatives on every trial, so one can discriminate between these two processes.
The SDMTS task also allows another interesting observation regarding perseveration. Each trial contains a forced choice of one of the three goal arms. If scopolamine were to increase perseveration of previously successful responses, then the accuracy of first choices should also be higher following treatment with centrally active scopolamine than under other conditions, because the rat would perseverate in returning to the location in which it had most recently been reinforced. Perseveration of this response would actually increase choice accuracy rather than decrease it. In contrast, the accuracy of both first and second choices declined following pretreatment with centrally active scopolamine.

The simultaneous examination of first-choice accuracy, second-choice accuracy, and the association between events on successive trials provides a powerful means to assess the nature of the memory deficit induced by scopolamine hydrobromide. The data from these experiments, along with those of Roitblat and Harley (1988; see also Roitblat, 1984b) suggest that there are two memory processes operating in support of SDMTS performance. The first of these is the encoding of the sample information. The second process involves discriminating the events of one trial from the events of previous trials. The present data imply that scopolamine interferes with memory performance in the SDMTS task by interfering with the strength or clarity of the memory code at the time the animal makes its choice (the encoding-failure hypothesis), rather than by decreasing the animal's ability to discriminate the events of one trial from the events of previous trials (the temporal discrimination hypothesis).

The analysis of the pattern of second choices following first-choice errors provides one means to assess the role of intrusions in determining choice accuracy. Second choices occurred only following first-choice errors, and the arm that was erroneously selected for the first choice was blocked off before the animal made its second choice. Second choices thus provide a relatively independent second assessment of the rats' memory for the sample. According to the temporal discrimination hypothesis, errors occur because two representationsone of the current sample and one from the previous trial-compete for control of the animal's choice. The occurrence of a first-choice error necessarily removes this source of competition because the same response is no longer available when the animal makes its second choice. If the effect of scopolamine were to increase intrusions from the previous trial, then first-choice accuracy may be suppressed by scopolamine, but second-choice accuracy should be enhanced because the choice associated with the competing representation is no longer available. In contrast, both experiments found that treatment with scopolamine decreased second-choice as well as firstchoice accuracy. This pattern of results indicates that errors occur, at least in part, because the animal has inadequate information about the identity of the correct choice. Second-choice errors (as well as first-choice errors) in- 
creased following treatment with scopolamine because scopolamine decreased the amount of information the animal had about the trial. First choices were based on inadequate information and the second choices that followed first-choice errors were similarly based on the same inadequate information. Apparently, the animals failed to encode and remember the location of the correct choice.

Sample agreement and increasing ITI durations affect separate mechanisms. Both rat (Roitblat \& Harley, 1988) and pigeon (Roitblat \& Scopatz, 1983) performance is better when two trials are separated by long as opposed to short ITI durations, even when the same sample is presented on both trials. Increasing ITI durations appears to affect both intrusions from the previous trial and encoding of the information from the present trial. Choice accuracy improves with longer ITI durations even when intrusions are not reduced or play no important role. In contrast, intertrial sample agreement can affect only intrusions. Together, they provide a means of assessing the relative roles of sample encoding and temporal discrimination (intrusions). Centrally active scopolamine appears to affect only one of these processes and not the other. Scopolamine seems to operate by reducing memory encoding rather than by increasing interference.

\section{REFERENCES}

Ammassari-Teule, M., \& CAPrioli, A. (1985). Spatial learning and memory, maze running strategies and cholinergic mechanisms in two inbred strains of mice. Behavioral Brain Research, 17, 9-16.

BEATTY, W. W., \& BIERLEY, R. A. (1985). Scopolamine degrades spatial working memory but spares spatial reference memory: Dissimilarity of anticholinergic effect and restriction of distal visual cues. Pharmacology, Biochemistry, \& Behavior, 23, 1-6.

Crow, T., \& Grove-White, I. (1973). An analysis of the leaming deficit following hyoscine administration in man. British Journal of Pharmacology, 49, 322-327.

DeUtsch, J. A. (1983). The physiological basis of memory. New York: Academic Press.

Drachman, D. A., Leavitt, J. (1974). Human memory and the cholinergic system: A relationship to aging? Archives of Neurology, 30, 113-121.

DunNeTt, S. B. (1985). Comparative effects of cholinergic drugs and lesions of nucleus basalis or fimbria fornix on delayed matching in rats. Psychopharmacology, 87, 357-363.

Eckerman, D. A., Gordon, W. A., Edwards, J. D., MacPhall, R. C., \& GAGE, M. L. (1979). Effects of scopolamine, pentobarbitol, and amphetamine on radial arm maze performance in the rat. Psychopharmacology, Biochemistry, \& Behavior, 12, 595-602.

Godding, P. R., Rush, J. R., \& Beatty, W. W. (1982). Scopolamine does not disrupt spatial working memory in rats. Pharmacology, Biochemistry, \& Behavior, 16, 919-923.

GRANT, D. S. (1981). Intertrial interference in rat short-term memory. Journal of Experimental Psychology: Animal Behavior Processes, 7, 217-227.
Heise, G. A., Hrabich, B., Lilie, N. L., \& Martin, R. A. (1975). Scopolamine effects on delayed alteration in the rat. Pharmacology, Biochemistry, \& Behavior, 00, 993-1002.

Heise, G. A., Hudson, J. D. (1985). Effects of pesticides and drugs on working memory in rats: Continuous delayed response. Pharmacology, Biochemistry, \& Behavior, 23, 591-598.

Huston, A. E., \& AGgleton, J. P. (1987). The effects of cholinergic drugs upon recognition memory in rats. Quarterly Joumal of Experimental Psychology, 39B, 297-314.

Kopelman, M. D. (1986). The cholinergic neurotransmitter system in human memory and dementia: A review. Quarterly Journal of Experimental Psychology, 38A, 535-573.

LeVy, H. D., KLuge, D. B., Elsmore, T. F. (1983). Radial arm maze performance of mice: Acquisition and atropine effects. $B e$ havioral \& Neural Biology, 39, 229-240.

OKAICHI, H., JARRARD, L. E. (1982). Scopolamine impairs performance of a place and cue task in rats. Behavioral \& Neural Biology, 35, 319-325.

ParrotT, A. C. (1987). Transdermal scopolamine: Effects of single and repeated patches upon psychological task performance. Neuropsychobiology, 17, 53-59.

RAUCH, S. L., \& RASKIN, L. A. (1984). Cholinergic mediation of spatial memory in the preweanling rat: Application of the radial arm maze paradigm. Behavioral Neuroscience, 98, 35-43.

RaWLINS, J. N. P. (1985). Associations across time: The hippocampus as a temporary memory store. Behavioral \& Brain Sciences, 8 , 479-496.

Rawlins, J. N. P., \& Olton, D. S. (1982). The septo-hippocampal system and cognitive mapping. Behavioral Brain Research, 5, 331-358.

RoItBlat, H. L. (1980). Codes and coding processes in pigeon shortterm memory. Animal Learning \& Behavior, 8, 341-351.

Roitblat, H. L. (1984a). Pigeon working memory: Models for delayed matching-to-sample performance. In M. L. Commons, A. R. Wagner, \& R. J. Herrnstein (Eds.), Quantitative analyses of behavior: Acquisition (Vol. 3, pp. 161-181). Cambridge, MA: Ballinger.

RoITblat, H. L. (1984b). Representations in pigeon working memory. In H. L. Roitblat, T. G. Bever, \& H. S. Terrace (Eds.), Animal Cognition (pp. 79-97). Hillsdale, NJ: Erlbaum.

RoITBLAT, H. L. (1987). Introduction to comparative cognition. New York: W. H. Freeman.

RoItBlat, H. L., \& HARLEY, H. E. (1988). Rat spatial delayed matchingto-sample performance: Acquisition and retention. Journal of $E x$ perimental Psychology: Animal Behavior Processes, 14, 71-82.

RoItBlat, H. L., \& Scopatz, R. A. (1983). Sequential effects in delayed matching-to-sample. Joumal of Experimental Psychology: Animal Behavior Processes, 9, 202-221.

Spencer, D. G., Pontecorvo, M. J., \& Heise, G. A. (1985). Central cholinergic involvement in working memory: Effects of scopolamine on continuous nonmatching and discrimination performance in the rat. Behavioral Neuroscience, 99, 1049-1065.

VisCARd, A. P., \& Heise, G. A. (1986). Effects of scopolamine on components of delayed response performance in the rat. Pharmacology, Biochemistry, \& Behavior, 25, 633-639.

Wirsching, B. A., Beninger, R. L., Jhamandas, K., Boegman, R. J., \& EL-Defrawy, S. R. (1984). Differential effects of scopolamine on working and reference memory of rats in the radial maze. Pharmacology, Biochemistry, \& Behavior, 20, 659-662.

(Manuscript received January 18, 1989; revision accepted for publication August 14, 1989.) 